\title{
Cyclopropanes and Hypervalent lodine Reagents: High Energy Compounds for Applications in Synthesis and Catalysis
}

\author{
Davinia Fernández González, Filippo De Simone, Jonathan P. Brand, Stefano Nicolai, and \\ Jérôme Waser*
}

\begin{abstract}
One of the major challenges faced by organic chemistry is the efficient synthesis of increasingly complex molecules. Since October 2007, the Laboratory of Catalysis and Organic Synthesis (LCSO) at EPFL has been working on the development of catalytic reactions based on the Umpolung of the innate reactivity of functional groups. Electrophilic acetylene synthons have been developed using the exceptional properties of ethynyl benziodoxolone (EBX) hypervalent iodine reagents for the alkynylation of heterocycles and olefins. The obtained acetylenes are important building blocks for organic chemistry, material sciences and chemical biology. The ring-strain energy of donor-acceptor cyclopropanes was then used in the first catalytic formal homo-Nazarov cyclization. In the case of aminocyclopropanes, the method could be applied in the synthesis of the alkaloids aspidospermidine and goniomitine. The developed methods are expected to have a broad potential for the synthesis and functionalization of complex organic molecules, including carbocycles and heterocycles.
\end{abstract}

Keywords: Acetylenes · Activated cyclopropanes $\cdot$ Catalysis $\cdot$ Hypervalent iodine $\cdot$ Synthesis

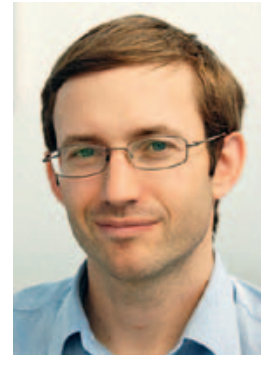

\section{Introduction}

In the last century, the achievements of organic chemistry made new drugs, materials and agrochemicals available to us, which have completely changed our life conditions. Despite these impressive achievements, organic synthesis is still facing important challenges in the future. With the awareness of the limited resources available on earth, the development of more efficient reactions is required. Furthermore, applications in life and material sciences are becoming increasingly sophisticated, demanding tailor-made complex organic molecules. In answer to these needs, the concepts of synthetic efficiency (atom and step economy) and 'green chemistry' have emerged. In particular, catalytic methods have been developed to replace stoichiometric reagents or to allow unprecedented bond disconnections.

\footnotetext{
${ }^{*}$ Correspondence: Prof. Dr. J. Waser

Ecole Polytechnique Fédérale de Lausanne Laboratory of Catalysis and Organic Synthesis EPFL SB ISIC LCSO

BCH 4306

$\mathrm{CH}-1015$ Lausanne

Tel.: +41216939388

Fax: + 41216939700

E-mail: jerome.waser@epfl.ch
}

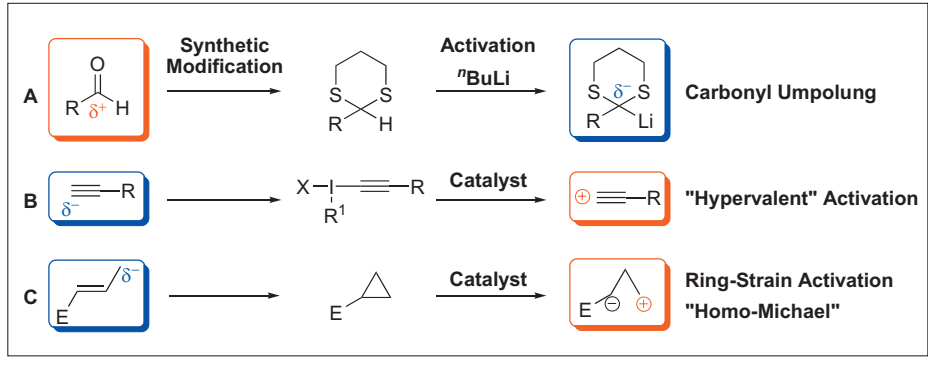

Scheme 1.

Umpolung of carbonyls, alkynes and Michael systems.

Since October 2007, the Laboratory of Catalysis and Organic Synthesis (LCSO) has focused its research in the discovery of catalytic reactions for non-classical bond disconnections. Our interest has been in bond formation requiring an inversion of the reactivity of functional groups (Umpolung). The Umpolung of functional groups is not a trivial task, and it is usually associated with a synthetic modification and an activation step (Scheme 1, A). Methods involving both catalytic modification and activation are still rare. A notable exception is the Umpolung of carbonyls using carbene catalysts and our contribution to this field resulted in the synthesis and investigation of the first bifunctional urea-carbene catalysts..$^{[1]}$

Our main focus of research is directed towards the Umpolung of functional groups which have been less widely investigated. In particular, we were interested in the development of electrophilic acetylene synthons (Scheme 1, B) and Michael systems electrophilic at the $\gamma$ position (Scheme 1, C). The importance of acetylenes in synthesis has grown exponentially in the last decades. Numerous catalytic processes using acetylenes have been introduced and they have been used extensively in material sciences and chemical biology. Conjugate addition on Michael systems is a fundamental reaction in organic chemistry. The corresponding homologous process is expected to be as useful, but has been much less investigated. In this short review, we will present our work on the Umpolung of acetylenes and Michael systems based on the use of hypervalent iodine reagents and cyclopropanes.

\section{Ethynyl Benziodoxolone (EBX) Reagents for the Umpolung of Acetylenes}

The higher electronegativity of sp hybridized carbons makes the deprotonation of alkynes to form acetylides easy, and consequently many reactions for the introduction of acetylenes into organic molecules are based on nucleophilic synthons. Electrophilic approaches are rare and have been mostly limited to halogen acetylenes, 
lead complexes and hypervalent iodine reagents. The first have low reactivity and the second are toxic, making hypervalent iodine reagents the most promising. ${ }^{[2]}$ Alkynyliodonium salts have been extensively investigated in the 80s and the 90 s in reactions with pre-formed salts and organometallic reagents. ${ }^{[3]}$ Convinced by their high potential, we decided to examine these reagents in catalytic processes, in particular the direct functionalization of $\mathrm{C}-\mathrm{H}$ bonds and olefins. However, the results obtained using the reported alkynyl iodonium salts were disappointing, as decomposition of these reagents was observed in presence of catalysts.

At this point, we became interested in the ethynylbenziodoxolone (EBX, 1)-based reagents described by Ochiai and Zhdankin, which are easily accessible in two steps on $30 \mathrm{~g}$ scale from 2-iodo benzoic acid. ${ }^{[4]}$ Similar reagents had been highly useful for trifluoromethylation reactions, ${ }^{[5]}$ but their use as acetylene transfer reagents in catalytic reactions had never been reported. The alkynylation of keto-esters 2 was examined first, and excellent results were obtained using TMS-EBX (1a) and fluoride sources (Scheme 2). ${ }^{[6]}$ The efficiency of acetylene transfer allowed us to extend the scope to acyclic keto- $(\mathbf{3 b}-\mathbf{d})$, cyano- (3e) and nitro- (3f,g) esters. The latter can be reduced in two steps into alkynyl amino-acid esters, which are not easily accessed using other methods.

We then decided to investigate the potential of EBX reagents for the alkynylation of electron-rich heterocycles (4, Scheme 3$)$. No reactivity was observed in the absence of catalyst, but formation of alkynylated indoles $(\mathbf{5 a}-\mathbf{i})$ and pyrroles $(\mathbf{5} \mathbf{j}-\mathbf{l})$ was obtained with $5 \mathrm{~mol} \% \mathrm{AuCl}$ and TIPS-EBX (1b). ${ }^{[7]}$ The developed method is highly practical, as it proceeds readily under air at room temperature using technical solvents. The reaction displayed an impressive functional group tolerance, including phenols $(\mathbf{5 c})$, acids (5d), nitro groups (5e), and halogens (5b) and is as such orthogonal to classical cross-coupling reactions. High selectivity for alkynylation of the most electron-rich position was observed. Recently, a one-pot process starting from unprotected $o$-alkynyl anilines and involving a $\mathrm{Au}(\mathrm{III})$-catalyzed cyclization followed by a $\mathrm{Au}(\mathrm{I})$-catalyzed alkynylation has also been developed. ${ }^{[8]}$

Thiophenes are much less nucleophilic than indoles and constituted a major challenge for our methodology. In fact, only traces of product were observed when using our developed conditions. However, the use of trifluoroacetic acid (TFA) as co-catalyst led to the formation of a broad range of alkynylated thiophenes $(\mathbf{5 m}-\mathbf{r}) .{ }^{[9]}$ In particular, less reactive conjugated aryl- and bi-thiophenes could also be alkynylated, which gave access to important

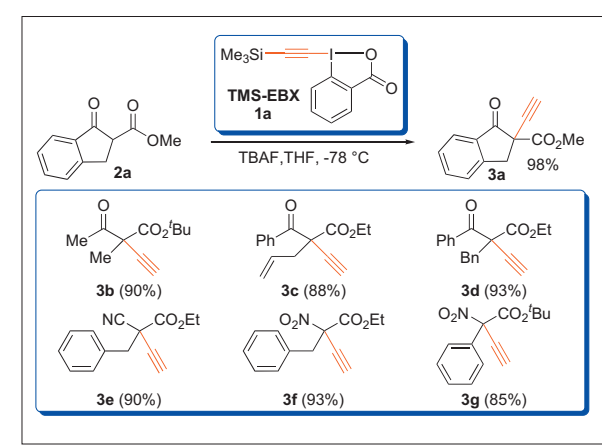

Scheme 2. $\alpha$-Alkynylation of activated carbonyls.

building blocks for material sciences. The elucidation of the reaction mechanism constitutes a formidable challenge. Two main pathways could be considered, the first involving redox active catalysis ( $\mathrm{Au}(\mathrm{I})$ to $\mathrm{Au}(\mathrm{III})$ ), and the second based on $\pi$ activation of the triple bond by gold. ${ }^{[7]}$

In another project, we were inspired by recent results reported in $\mathrm{Pd}-$ catalyzed cyclization of nucleophiles onto non-activated olefins followed by oxidative $\mathrm{C}-\mathrm{X}$ bond formation. ${ }^{[10]}$ The related oxy- and amino- alkynylation reactions had never been reported. We were pleased to see that oxyalkynylation of olefins was possible with TIPS-EBX and phenols or carboxylic acids as nucleophiles (Scheme 4, products 7a-f). ${ }^{[11]}$ The extension of the reaction to the synthesis of lactams was not an easy task, as only very low yields were obtained using $\operatorname{Pd}(\text { hfacac })_{2}$ (hfacac = hexafluoroacetylacetonate) as catalyst. Fortunately, the use of in situ formed palladate complexes was highly successful, allowing the synthesis of not only $\delta$-lactams $(\mathbf{7 g}, \mathbf{h})$, but also oxazolidinones (7i), imidizadolidinones (7j) and $\delta$-lactams $(\mathbf{7 k}, \mathbf{l})$ heterocycles. ${ }^{[12]}$ The methodology was applied in the 9-steps synthesis of ( \pm )-trachelanthamidine (13), a pyrrazolidine alkaloid (Scheme 5).

\section{Cyclization Reactions of Activated Cyclopropanes}

Cyclopropanes, especially when donor-acceptor substituted are extremely useful reactive intermediates in organic chemistry.[13] They can be considered as homologous of olefins with a partial $\pi$ bond character (Walsh orbitals). Electrocyclization reactions on the other hand are important reactions for the synthesis of carbocycles, and the Nazarov cyclization of divinyl ketones is especially useful for the synthesis of cyclopentenones. Substitution of one of the vinyl groups by a cyclopropane leads to the corresponding formal homo-Nazarov reaction, which had been reported only with few substrates using large excess of Lewis or Brønsted acids in the past. ${ }^{[14]} \mathrm{We}$ developed the first catalytic homo-Nazarov reaction of cross-polarized substrates bearing an electron-rich aromatic ring on the cyclopropane and a heteroatom on the double bond (Scheme 6). ${ }^{[15]}$

In view of applications in the synthesis of natural products, we wondered if a nitrogen heteroatom could also play the role of activating group on the cyclopropane. Such intermediates could in principle give a general entry to the aspidosperma class of natural products, such as aspidospermidine (14) (Scheme 7). The required aminocyclopropane $\mathbf{1 6}$ could be accessed in seven steps from $\delta$-valerolactam (17). ${ }^{[16]}$ The projected cyclization reaction was indeed highly successful, but a mixture of regioisomers resulting from attack on $\mathrm{C}(3)$ (18) or $\mathrm{N}(1)$ (19) was initially obtained. Fortunately, it was possible to control the regioselectivity of the reaction by

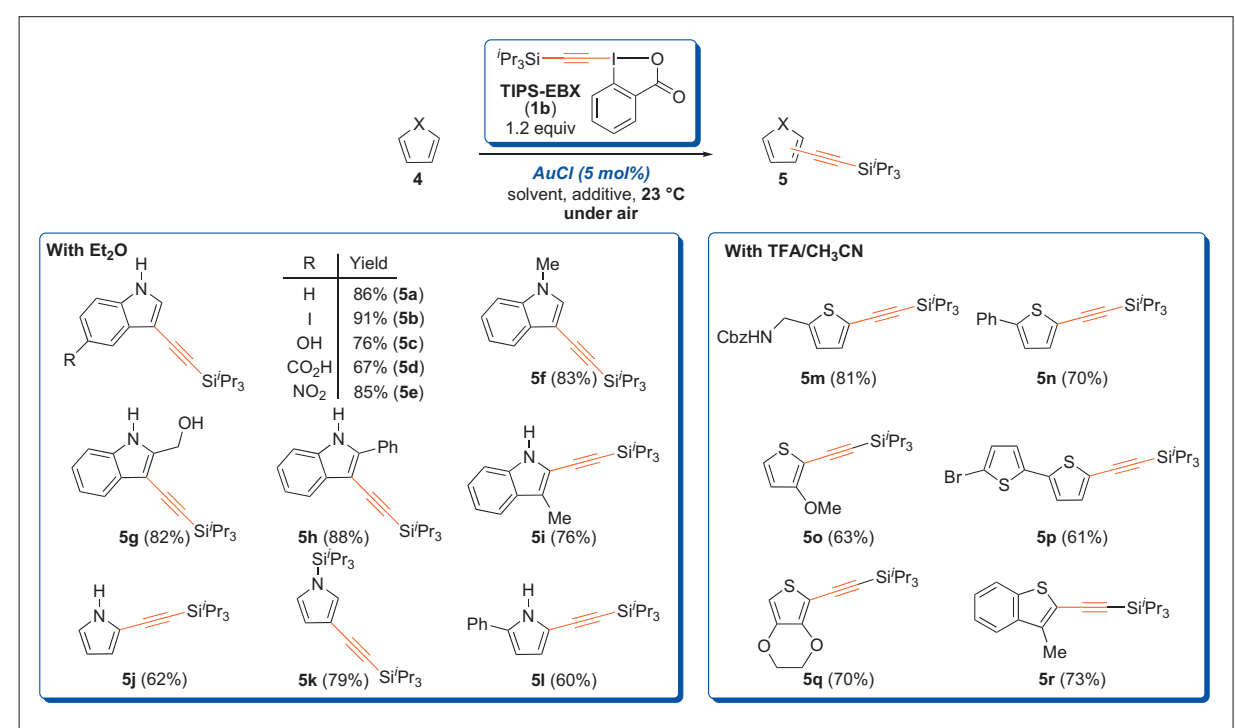

Scheme 3. Alkynylation of indoles, pyrroles and thiophenes. 


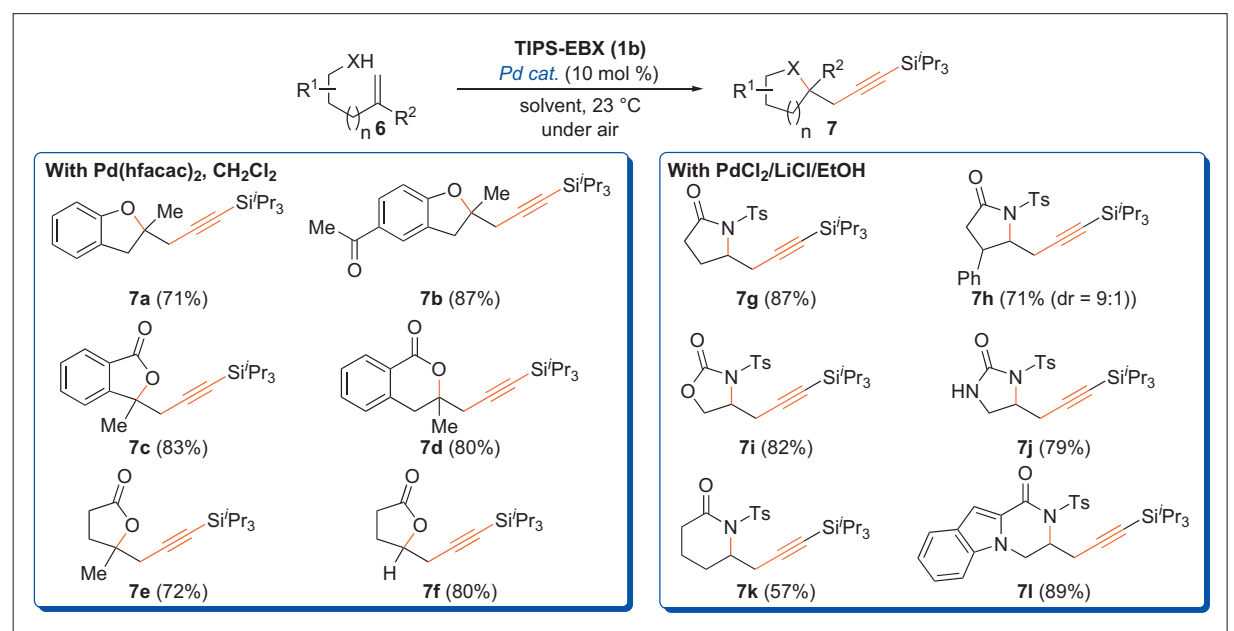

Scheme 4. Oxy- and amino-alkynylation of olefins.

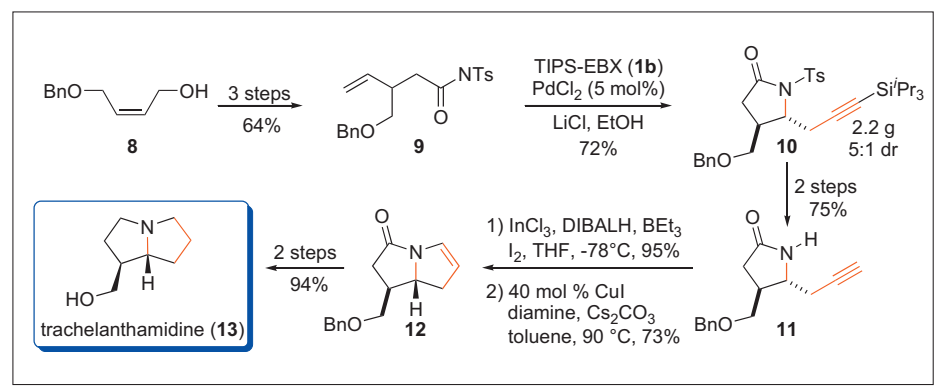

Scheme 5. Total synthesis of $( \pm)$-trachelanthamidine.

the choice of catalyst and solvents: copper catalysts in acetonitrile led predominantly to $\mathbf{1 8}$, whereas toluene sulfonic acid in dichloromethane gave $\mathbf{1 9}$. Both products are highly useful: deprotection of $\mathbf{1 8}$ to give $\mathbf{1 5}$ led to a formal synthesis of aspidospermidine (14), based on the work of Wenkert,[17] whereas 19 contains the polycyclic scaffold of goniomitine (22), a rare natural product isolated from gonioma malagasy. The synthesis of this natural product could be accomplished in five steps only from 3-substituted indole derivative 20. ${ }^{[16]}$ With significant amounts of goniomitine available, the first investigation of the bioactivity became possible, which revealed a modest antiproliferative effect $(29-73 \mu \mathrm{Mol})$.

\section{Conclusion}

Since January 2008, research at LCSO has been focused on the use of small, highenergy intermediates for the development of non-classical bond formations under catalytic conditions. EBX reagents have been introduced as excellent electrophilic acetylene transfer reagents for alkynylation reactions under metal-free and metal-catalyzed $(\mathrm{Pd}, \mathrm{Au})$ conditions. The reactivity of cyclopropanes has been exploited in the further development of the formal homoNazarov reaction. Both reactions have given access to important building blocks and have already been applied in the synthesis of alkaloid natural products. The potential
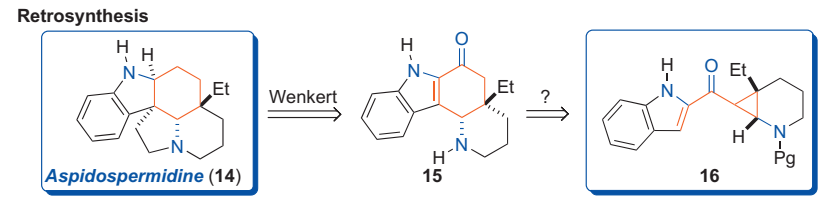

Key Step
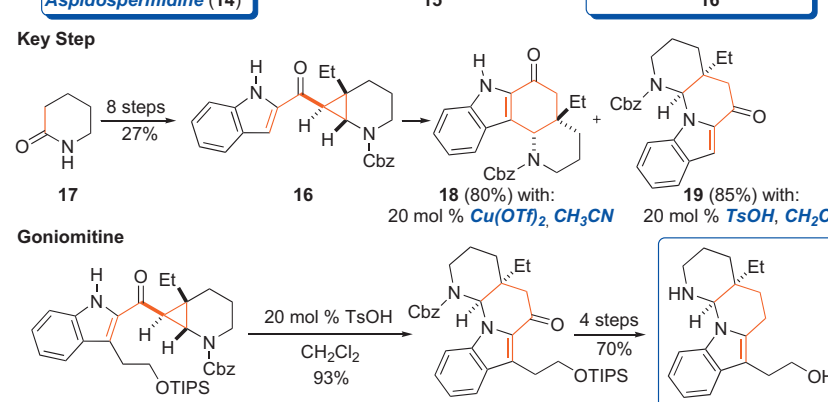

Scheme 7.

Cyclization of aminocyclopropanes and alkaloids synthesis.

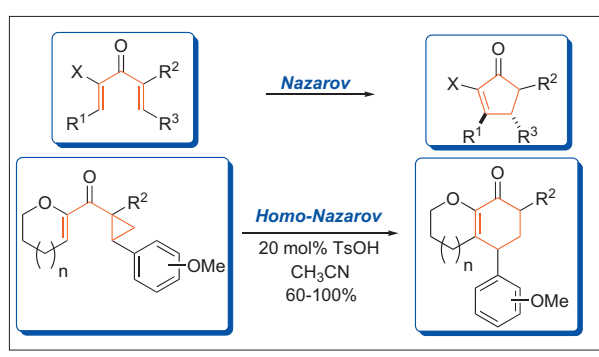

Scheme 6. Nazarov and homo-Nazarov reactions.

of the new reactivity discovered has just begun to be revealed, and a broad potential for the synthesis and functionalization of complex organic molecules, including carbocycles and heterocycles can be expected in the future.

\section{Acknowledgments}

EPFL and SNF (grant number 200021 119810) are acknowledged for financial support. We thank F. Hoffmann-La Roche Ltd for an unrestricted research grant.

Received: May 27, 2011

[1] J. P. Brand, J. I. O. Siles, J. Waser, Synlett 2010 881.

[2] V. V. Zhdankin, P. J. Stang, Chem. Rev. 2008, $108,5299$.

[3] V. V. Zhdankin, P. J. Stang, Tetrahedron 1998 $54,10927$.

[4] a) M. Ochiai, Y. Masaki, M. Shiro, J. Org. Chem. 1991, 56, 551; b) V. V. Zhdankin, C. J. Kuehl, A. P. Krasutsky, J. T. Bolz, A. J. Simonsen, J. Org. Chem. 1996, 61, 654; c) J.P. Brand, D. Fernández González, S. Nicolai, J. Waser, Chem. Commun., 2011, 47, 102. TIPSEBX (1b) is now available from Sigma-Aldrich

[5] a) I. Kieltsch, P. Eisenberger, A. Togni, Angew. Chem., Int. Ed. 2007, 46, 754; b) R. Koller, K. Stanek, D. Stolz, R. Aardoom, K. Niedermann, A. Togni, Angew. Chem., Int. Ed. 2009, 48, 4332.

[6] D. Fernández González, J. P. Brand, J. Waser, Chem. Eur. J. 2010, 16, 9457.

[7] J. P. Brand, J. Charpentier, J. Waser, Angew. Chem., Int. Ed. 2009, 48, 9346.

[8] J. P. Brand, C. Chevalley, J. Waser, Beilstein J. Org. Chem. 2011, 7, 565.

[9] J. P. Brand, J. Waser, Angew. Chem., Int. Ed. 2010, 49, 7304.

[10] A. Minatti, K. Muniz, Chem. Soc. Rev. 2007, $36,1142$.

[11] S. Nicolai, S. Erard, D. Fernández González, J. Waser, Org. Lett., 2010, 12, 384.

[12] S. Nicolai, C. Piemontesi, J. Waser, Angew Chem., Int. Ed. 2011, 50, 4680

[13] a) H. U. Reissig, R. Zimmer, Chem. Rev. 2003 , 103, 1151; b) F. De Simone, J. Waser, Synthesis 2009, 3353 .

[14] a) W. S. Murphy, S. Wattanasin, Tetrahedron Lett. 1980, 21, 1887; b) O. Tsuge, S. Kanemasa, T. Otsuka, T. Suzuki, Bull. Chem. Soc. Jpn. 1988, 61, 2897; c) F. De Simone, J. Waser, Chimia 2009, 63, 162.

[15] F. De Simone, J. Andres, R. Torosantucci, J. Waser, Org. Lett. 2009, 11, 1023.

[16] a) F. De Simone, J. Gertsch, J. Waser, Angew. Chem., Int. Ed. 2010, 49, 5767; b) F. De Simone, J. Gertsch, J. Waser, Synlett 2011, 589.

[17] E. Wenkert, T. Hudlicky, J. Org. Chem. 1988, 53, 1953. 